\title{
Lessons of dislocation
}

\section{Daniel Imhoff ${ }^{1}$}

Accepted: 25 April 2020 / Published online: 18 May 2020

(c) Springer Nature B.V. 2020

\section{Introduction}

On a Saturday morning in early March I arrived at the local farm supply hoping to buy two dozen baby chicks. Just five minutes after opening I found myself number 46 in a queue that wrapped from the store front across the parking lot and around the corner. Blue tape marks were spaced every six feet on the pavement but few people observed the social distancing guidelines recently issued in Sonoma County.

This scene freaked me out. In addition to hoarding cleaning and personal hygiene products - our collective survival instinct was to become personally involved in the food supply. Never mind that the chicks would take at least five months to lay an egg. After an hour I reached the incubation room. Only three of the original fifteen varieties remained. But the Cornish Cross broilers I wanted had barely been selected. In eight weeks these chicks would reach harvest weight. I was determined that they would be raised as healthfully as possible: with sunshine, fresh bedding, organic feed twice a day, and grapefruit seed extract to cleanse their fragile digestive systems.

By mid-April a New York Times article (Corkery and Yaffe-Bellany 2020) reported that meat processing factories had become hot spots for Coronavirus outbreaks. Decades of consolidation have created bottlenecks in our nation's food system and meat processing is a glaring example. Since the 1980s there has been a broad scale shift from unionized plants in urban centers to rural areas close to feedlots and cheap immigrant labor pools. Only 800 slaughterhouses in the United States now process billions of pounds of beef, pork and chicken per year. They are massive and geographically concentrated. Work on these disassembly lines is insanely fast-aced, grueling and dangerous. Smaller-scale

This article is part of the Topical Collection: Agriculture, Food \& Covid-19.

Daniel Imhoff

danimhoff@mac.com

1 Watershed Media, Healdsburg, USA regionally based facilities have all but disappeared, making it increasingly difficult every year for independent livestock farmers to survive.

Concern was rising about a potential meat shortage if these massive slaughter factories temporarily closed or even slowed down. The New York Times article profiled one South Dakota Smithfield pork processing plant that exhibited a 16 percent COVID-19 infection rate among its 3700 workers. It was uncertain whether this abnormally high infection rate was the result of the state's voluntary social distancing guidelines or the close proximity of workers on the disassembly lines. That single plant processes 5 percent of the nation's pork.

Threats to food system workers weren't limited to slaughter facilities. Workers in grocery stores, restaurants, and all throughout delivery chains found themselves on the front lines as well. Meanwhile, with the downturn in commercial markets, the slowdown of meat packing, and a dearth of labor, farmers were dumping milk, culling livestock and allowing vegetables to rot.

Animal factories have been a serious public health issue since the mid nineteenth century. But this crisis has harshly illuminated the severe costs of the monopolization and industrialization and simplification of our food system: the devastation of rural communities, the loss of regional production infrastructure, the war on nature that impacts air and fresh water, countless imperiled species and vanishing habitats.

Each year Congress directs tens of billions of US taxpayer dollars to compensate farmers for inevitable losses through the Farm Bill. This helps to ensure an oversupply of corn and soybeans and other commodities that are mainly fed to livestock, manufactured into ethanol or exported. Meanwhile $70 \%$ of Americans are overweight or obese, a significant risk factor for Coronavirus.

I fear this current pandemic may simply be a dress rehearsal, just one crisis in the midst of a long emergency that is going to reshape life as we know it. We can no longer afford to squander time, energy or resources building a food 
system that is so big and so vulnerable to contamination and dislocation that it will inevitably fail us.

Keeping backyard gardens with laying hens is something all of us who can absolutely should do. But the time is now to rebuild and re-envision a modern concept of food security. Smaller scale, regionally diversified production as well as restoring a healthy relationship between humans and the natural world should be our top priorities.

Daniel Imhoff is an author and musician who lives in northern California. His books include The Farm Bill: A Citizen's Guide, CAFO: The Tragedy of Industrial Animal Factories and Farming with the Wild: Enhancing Biodiversity on Farms and Ranches.

\section{Reference}

Michael Corkery and David Yaffe-Bellany. April 18, 2020. The Food Chain's Weakest Link: Slaughterhouses. NYT, web edition.
Publisher's Note Springer Nature remains neutral with regard to jurisdictional claims in published maps and institutional affiliations.

Daniel Imhoff is an author and musician who has been writing about agriculture, conservation, design and public policy for more than 25 years. His many books include The Farm Bill: A Citizen's Guide, CAFO: The Tragedy of Industrial Animal Factories (winner of the 2011 Nautilus Gold Prize for Investigative Reporting), and Farming with the Wild: Enhancing Biodiversity on Farms and Ranches. He lives on a homestead farm in Northern California. 